\title{
CONSECUENCIAS DEL ESTRÉS CALÓRICO SOBRE LA REPRODUCCIÓN DEL GANADO VACUNO
}

\section{CONSEQUENCES OF HEAT STRESS ON REPRODUCTION OF CATTLE}

Córdova Izquierdo, A. ${ }^{1}$, Iglesias Reyes, A.E. ${ }^{1}$, Ruiz Lang, C.G. ${ }^{1}$, Guerra Liera, J.E. ${ }^{2}$, Inzunza Castro, J.F. ${ }^{2}$, Villa Mancera, E.A. ${ }^{3}$, Juárez Mosqueda, M.L. ${ }^{4}$, Gómez Vázquez, A. ${ }^{5}$, Cansino Arroyo, G. ${ }^{5}$, Olivares Pérez, J. ${ }^{6}$, Espinosa Cervantes, R. ${ }^{1}$ y Velázquez Ordóñez, V. ${ }^{7}$

${ }^{1}$ Departamento de Producción Agrícola y Animal. Universidad Autónoma Metropolitana Unidad Xochimilco, México, D.F. acordova@correo.xoc.uam.mx. ${ }^{2}$ Facultad de Agronomía. Universidad Autónoma de Sinaloa, México. ${ }^{3}$ Facultad de Veterinaria. Benemérita. Universidad autónoma de Puebla, México. ${ }^{4}$ FMVZ-UNAM. ${ }^{5}$ División Académica de Ciencias Agropecuarias. Universidad Juárez Autónoma de Tabasco, México. ${ }^{6}$ Unidad Académica de Veterinaria. Universidad Autónoma de Guerrero, México. ${ }^{7}$ Centro de Investigación y Estudios Avanzados en Salud Animal. FMVZ-UAEM.

\section{RESUMEN}

El estrés calórico representa unos de los principales factores del medio ambiente que repercuten negativamente en el desempeño reproductivo del ganado vacuno. En este trabajo se describen los ajustes conductuales, mecanismos físicos y respuestas fisiológicas para la pérdida calórica; ventajas adaptativas del bos indicus con respecto al bos Taurus, fisiopatología del estrés calórico y repercusiones del estrés calórico en la reproducción animal, tanto en el macho como en la hembra.

PALABRAS CLAVE: Estrés calórico, ganado vacuno, reproducción. 


\begin{abstract}
Heat stress represents one of the major environmental factors that adversely affect the reproductive performance of cattle. In this paper the behavioral adjustments, physical mechanisms and physiological responses to heat loss are described; bos indicus adaptive advantages with respect to bos Taurus, pathophysiology of heat stress and heat stress effects in animal reproduction, both the male and the female.
\end{abstract}

KEY WORDS: Heat stress. Cattle, breeding.

\title{
INTRODUCCIÓN
}

La vida depende de la energía y el organismo animal es un transformador de energía que es convertida en actividad física como locomoción, crecimiento, mantenimiento, reproducción e incluso en la obtención de productos útiles como lana y leche. Esta energía la obtienen a través de la acción química de los nutrientes de la ingesta, además de la energía radiante del sol.

La fisiología climática es un área que aporta conocimientos muy relevantes en el manejo de los animales expuestos a calor. El término intercambio calórico implica tanto la pérdida calórica desde el animal hacia el ambiente, o en caso opuesto, la ganancia de calor por el animal. Estos intercambios de calor son denominados "sensibles", ya que la pérdida de calor es una forma de energía que puede ser detectada por los receptores sensoriales, por ejemplo, en condiciones ambientales donde hay altas temperaturas, los animales tienden a incrementar su temperatura corporal y para regularla existe una perdida calórica por evaporación, ya sea por sudoración, jadeo o polipnea térmica.

En el caso de la temperatura corporal de los poiquilotermos o exotermos (reptiles, anfibios y peces) fluctúa directamente con la temperatura ambiental, en estas especies la fuente primaria de calor es externa, es decir, si necesitan enfriar su cuerpo deben desplazarse a un ambiente más fresco, a este tipo de termorregulación se llama conductual. Existe otro tipo de obtención de energía en los animales que reciben el nombre de homeotermos o endotermos, y comprende a la mayoría de aves y mamíferos, los cuales son capaces de elaborar respuestas para producir o conservar el calor.

Cuando los animales se encuentran en un rango de temperatura intermedia (sin calor o sin frío) es fácil para ellos mantener su temperatura corporal, a este rango se le llama zona termoneutral y varía en cada especie. En esa zona la producción de calor en el organismo animal es mínima; pero en temporadas de calor cuando la temperatura ambiental se eleva, se dice que 
el animal se encuentra en una temperatura crítica, ya que la temperatura corporal del animal se incrementa debido a que el calor no se puede liberar de forma eficiente, incrementando el consumo de oxígeno en el animal (Fowler, 2008; Fowler, 2006).

\section{AJUSTES CONDUCTUALES, MECANISMOS FÍSICOS Y RESPUESTAS FISIOLÓGICAS PARA LA PÉRDIDA CALÓRICA}

A lo largo del tiempo los animales han adoptado ciertos comportamientos para poder regular más fácil la temperatura corporal, por ejemplo, ante el calor los bovinos extienden sus extremidades para facilitar los mecanismos de pérdida calórica, también aquellos bovinos que están en zonas desérticas buscan las sombras disponibles y pastorean en las horas más frescas de la mañana y de la tarde, otra conducta termorregulatoria ante el calor que han adoptado los bovinos, es separarse entre ellos o mantenerse alejados unos de otros.

Otras características muy importantes a tomar en cuenta son las adaptaciones morfológicas que han sufrido ante el calor las diferentes especies animales, entre estas están el incremento de tamaño de las orejas, ya que teniendo una mayor superficie auricular permite un mayor enfriamiento por evaporación, además también la conducción, la convección y la radiación se incrementan, también el movimiento de las orejas favorece la disipación calórica. Otra de las características en el caso de los bovinos son los cuernos, se ha demostrado que los cuernos tienen participación en la termorregulación

Entre las respuestas fisiológicas para la perdida calórica (termodisipación) que tienen los animales se puede destacar las respuestas cutáneas, en las que se incluyen: la vasodilatación y la respuesta respiratoria.

La vasodilatación ocurre cuando los vasos cutáneos se dilatan existiendo variaciones de la sangre periférica que fluye por la piel, alterando la temperatura cutánea, que favorece la pérdida calórica por la piel, en caso de que la temperatura del medio se eleve más allá del nivel crítico, esta vasodilatación se incrementa, aumentando la sudoración que es promovida por las fibras simpáticas colinérgicas que inervan a las glándulas écrinas de la piel. La evaporación cutánea se produce en todas las especies (perdida de calor insensible), siempre y cuando la humedad relativa ambiental no sea muy alta e impida que se agregue más vapor de agua al ambiente. En las especies con abundantes glándulas sudoríparas en la piel, como por ejemplo el caballo, mantiene un mecanismo de enfriamiento eficiente condicionado a que la ingesta de agua sea suficiente. 
Las respuestas respiratorias, que es otro tipo de enfriamiento evaporativo, se lleva a cabo en los pulmones a través de la espiración; al haber una mayor temperatura ambiental se activa el mecanismo respiratorio de espiración, promovido por el centro espiratorio del bulbo raquídeo y el centro neumotáxico de la protuberancia anular o puente de Varolio. Algunas especies como por ejemplo el perro, jadean, lo cual incrementa el enfriamiento evaporativo en el tracto respiratorio. En los ungulados la respiración con la boca abierta puede ayudar a disipar el calor;

Otra respuesta conductual adoptada a las altas temperaturas externas, es activarse los mecanismos fisiológicos como la respuesta cardiovascular y la baja en el consumo voluntario de alimento, entre otras, y metabólicos que son consecuencia de la secreción hormonal, de las cuales las más reportadas es la referida a glucocorticoesteroides. Ambas respuestas tienen como objetivo el mantener el balance calórico del animal. Pero si estas respuestas no son suficientes para regular la temperatura interna del animal, este llega al fallo termorregulatorio que puede provocar la muerte del animal debido a la hipertermia, modificaciones den el balance-equilibrio hidromineral y acido básico (Yokoyama et al., 2004; Blecha, 2000).

\section{VENTAJAS ADAPTATIVAS DEL Bos indicus CON REPECTO AL Bos Taurus}

En la Tabla 1, se muestra que la capa del Bos indicus tiene mayor reflexión que la del Bos taurus. Lo cual le da al primero una ventaja adaptativa para resistir la incidencia de los rayos infrarrojos. En relación a enfriamiento evaporativo el ganado presenta una mayor capacidad de sudoración que el ganado europeo, por lo tanto este último necesita perder mayor cantidad de calor por vía respiratoria.

En el tema de conservación de agua tanto el ganado asiático cómo el europeo, tienen una conservación ligeramente similar, pues el ganado Bos indicus posee un respuesta más eficiente a la secreción de vasopresina, esto hace que pueda reabsorber mayor cantidad de agua en los túbulos contorneados distales y túbulos colectores de la nefrona. En el caso de ganado asiático, la conservación de agua es más eficiente en el tracto intestinal.

En tanto color de piel del ganado, en el asiático es negro, haciendo que estos animales resistan mejor la exposición prolongada a los rayos ultravioleta del sol. Sin embargo otras especies que aparecen presentan una mejor capacidad adaptativa al calor (Fowler, 2006) 
Tabla 1. Rumiantes expuestos al calor y a la insolación durante el verano en trópico seco y húmedo. En la tabla se presentan distintos tipo de capa, mecanismos de enfriamiento y niveles de conservación de agua.

\begin{tabular}{|c|c|c|c|c|c|c|c|}
\hline & \multicolumn{2}{|c|}{ Capa o abrigo } & \multicolumn{2}{|c|}{$\begin{array}{l}\text { Enfriamiento } \\
\text { evaporativo }\end{array}$} & \multicolumn{2}{|c|}{$\begin{array}{l}\text { Conservación } \\
\text { de agua }\end{array}$} & \multirow[b]{2}{*}{$\begin{array}{l}\text { Color } \\
\text { de la } \\
\text { piel }\end{array}$} \\
\hline & $\begin{array}{l}\text { Reflexió } \\
\text { n }\end{array}$ & $\begin{array}{l}\text { Aislamient } \\
\text { o }\end{array}$ & $\begin{array}{l}\text { Respiratori } \\
\text { o }\end{array}$ & $\begin{array}{l}\text { Sudoració } \\
\text { n }\end{array}$ & Renal & Fecal & \\
\hline $\begin{array}{l}\text { Borrego } \\
\text { merino }\end{array}$ & + & +++ & +++ & + & ++ & ++ & Blanco \\
\hline $\begin{array}{l}\text { Borrego } \\
\text { peliguey }\end{array}$ & ++ & ++ & ++ & ++ & ++ & ++ & $\begin{array}{l}\text { Obscur } \\
\text { o }\end{array}$ \\
\hline Cabra & ++ & + & ++ & ++ & ++ & ++ & $\begin{array}{l}\text { Obscur } \\
\text { o }\end{array}$ \\
\hline $\begin{array}{l}\text { Bos } \\
\text { taurus } \\
\text { Shorthor } \\
n\end{array}$ & ++ & + & ++ & ++ & + & + & Blanco \\
\hline $\begin{array}{l}\text { Bos } \\
\text { indicus } \\
\text { Bibos } \\
\text { banteng }\end{array}$ & +++ & \pm & + & +++ & + & ++ & Negro \\
\hline Búfalo & - & - & + & +++ & + & + & Negro \\
\hline
\end{tabular}

$(+++)$ Mayor efecto, alta eficiencia; $(++)$ moderada actividad; $(+)$ algún efecto; $( \pm)$ muy poca actividad; (-) ninguna actividad. Modificado de Fowler, 2006

\section{FISIOPATOLOGÍA DEL ESTRÉS CALÓRICO}

La hipertermia es una elevación excesiva de la temperatura corporal. Las principales causas de esta alteración son principalmente la prolongada exposición a temperaturas ambientales elevadas, humedad elevada, deshidratación o deficiente consumo de agua, ya que si la temperatura corporal del animal se eleva la necesidad de ingesta de agua también será mayor; otra causa importante de hipertermia es el excesivo trabajo muscular, por lo cual el 
manejo de los animales en épocas de calor debe ser limitado. También el manejo inadecuado de microclima (deficiente ventilación) conduce a una hipertermia.

La hipertermia incrementa la actividad metabólica y el consumo celular de oxígeno (se incrementa $10 \%$ por cada grado $\mathrm{C}$ en seres humanos). En mamíferos cuando la temperatura corporal excede $\operatorname{los} 41^{\circ} \mathrm{C}$, la necesidad de oxígeno excede la cantidad de oxígeno que puede suministrar la respiración normal, lo cual puede llevar al individuo a un daño celular por hipoxia. El encéfalo, el hígado y los riñones son los órganos más afectados (Moberg,1995).

Los efectos de la hipertermia en los sistemas orgánicos pueden ser graves, sobre todo si el estrés calórico es severo y prolongado. El sistema nervioso central (SNC) es el más sensible a la hipertermia, es frecuente encontrar necrosis de neuronas, y también como efecto secundario a la hipotensión e hipoxia cerebral. La elevación de la temperatura corporal provoca un reacomodo sanguíneo desde las vísceras hacia la piel. El riego sanguíneo disminuido en el tracto gastrointestinal provoca inhibición de la función digestiva. La hipovolemia provoca frecuentemente un decremento de la función de la filtración glomerular y una insuficiencia renal (uremia pre-renal).

La hipertermia también provoca hemoconcentración, desbalance electrolítico, fragilidad de los eritrocitos y leucocitosis, la cuenta de plaquetas disminuye y la cascada de la coagulación se altera de forma severa e incluso letal (Blecha, 2000).

La hipertermia incrementa la actividad metabólica y el consumo celular de oxigeno (10 por ciento por cada grado $\left.{ }^{\circ} \mathrm{C}\right)$. En los mamíferos cuando la temperatura corporal supera los $41^{\circ} \mathrm{C}$, el consumo de oxigeno requerido excede al obtenido por la respiración normal (eupnea) y esto conduce a un daño hipóxico celular. El encéfalo, el hígado y los riñones son los órganos más sensibles a ese daño.

Los efectos de la hipertermia en los diversos órganos y sistemas pueden ser profundos, en el caso de que el estrés calórico sea severo y prolongado, puede repercutir en la función de varios órganos e incluso llegar a producir la muerte del animal.

El sistema nervioso central (SNC) es el más sensible a la hipertermia. Los efectos del calor pueden producir necrosis en las neuronas, o este mismo efecto se puede presentar por factores secundarios como la hipotensión, que provoca hipoxia cerebral o producir efectos en los sistemas cardiovascular y sanguíneo (hemorragia, coágulos intravasculares diseminados, que provocan lesiones en el SCN). También la hipertermia puede provocar hemoconcentración, desbalance electrolítico, fragilidad aumentada de los eritrocitos, leucocitosis y alcalosis respiratoria causada por la elevada eliminación de $\mathrm{CO} 2$ durante la polipnea térmica (Fowler, 2008). 


\section{REPERCUSIONES DEL ESTRÉS CALÓRICO EN LA REPRODUCCIÓN ANIMAL}

Dobson y Smith (2000), indicaron que el proceso de la reproducción es un sistema fisiológico muy importante para el desarrollo de las especies, ligado al estrés, que Coubrough (1985), lo ha clasificado en dos grupos: estrés ambiental y estrés por manejo. El estrés ambiental incluye a la temperatura del ambiente, al frío y/o frío calor, al viento y a la humedad. El estrés por manejo incluye a la densidad animal, a los procedimientos de manejo, al flujo de animales, a la interacción entre animales de la misma o diferente especie y la condición social existente, como: angustia psicológica inespecífica, ruido; trauma físico, etc. La combinación de ambos tipos de estrés, actúan como estresantes, lo cual compromete a la homeostasis del animal. Por otro lado, Dobson and Smith (1995), mencionaron los siguientes tipos de estrés: físico, que incluye al transporte de animales y al daño físico; psicológico, en el cual se contempla el aislamiento de los animales; fisiológico, que se considera a la hipoglucemia y a los cambios en la presión sanguínea, aspectos todos relacionados con el proceso reproductivo de los mamíferos.

El mantenimiento constante de la temperatura corporal normal se conoce como homeotermia y es de importancia vital, para el funcionamiento de los tejidos encefálicos. En el bovino, la variación diurna normal en la temperatura corporal debe ser de 0.6 a $1.2^{\circ} \mathrm{C}$. Un aumento mayor a $1.2^{\circ} \mathrm{C}$ es signo de enfermedad o de mala adaptación a la elevación térmica. Este rango de temperatura se conoce como zona de confort y varía de acuerdo a la especie y a las razas de animales. Cuando los animales tienen a una temperatura corporal inferior o superior a su grado de confort, el ritmo metabólico disminuye o aumenta de lo normal, presentándose un desequilibrio en el bienestar animal. El calor o temperatura corporal, puede ser eliminado mediante los siguientes mecanismos: radiación, conducción, convección y evaporación (Yabuta, 2000).

A continuación se describen algunas repercusiones del estrés calórico sobre la reproducción en el macho y la hembra.

\section{EN EL MACHO}

Calidad seminal: El estrés ambiental puede provocar baja calidad seminal, la cual está íntimamente relacionada con la baja fertilidad, debido probablemente a una combinación de bajas tasas de fertilización. Los testículos al estar suspendidos en el escroto, la espermatogénesis es afectada al exponerse a altas temperaturas en el exterior, lo que provoca 
baja calidad espermática, lo cual también está directamente relacionado con la calidad del eyaculado (Nezhad et al., 2013; Wechalekar et al., 2010; Rodríguez, 2007; Coubrough, 1985; Chemineau, 1993). Esta baja calidad seminal es debida principalmente a las afectaciones que sufren las células de Sertoli por el estrés calórico; este a su vez induce apoptosis, estrés oxidativo en dichas células, el cual puede inducir a la infertilidad por el daño que ocasiona en los lípidos y proteínas de la membrana del espermatozoide, también por el daño que provoca en el ADN del espermatozoide, esto se traduce a un pobre desarrollo embrionario y abortos involuntarios (Nezhad et al., 2013; Aitken y De Luliis, 2010; Tremellen, 2008; Jung y Schuppe, 2007). Estudios recientes realizados por Rutledge (2001), sugirieron que el efecto del estrés sobre la calidad de los espermatozoides, puede mejorarse con la puesta en marcha de la tecnología de la congelación seminal; sin embargo, el útero de las hembras, pueden representar estrés térmico para los espermatozoides.

\section{EN LA HEMBRA}

El comportamiento sexual y la tasa de fertilidad, son los principales indicadores en la reproducción de las hembras mamíferas que se afecta negativamente por el estrés ambiental. De tal manera que los programas emprendidos con el fin de aumentar la fertilidad de las hembras domésticas, tienen menor éxito en las épocas calurosas que en las templadas (Chemineau, 1993).

El estrés por calor compromete el eje hipotálamohipófisis, afectando la pulsatilidad de las gonadotropinas, lo cual a su vez incide de manera negativa sobre la expresión de signos que hagan evidente el celo al ocasionar alteraciones sobre el crecimiento folicular y conduciendo a la inhibición del desarrollo embrionario. En vacas de la raza Holstein expuestas al estrés por calor, con temperaturas superiores a $\operatorname{los} 29^{\circ} \mathrm{C}$ durante 20-50 días antes de la inseminación artificial, se observaron tasas de gestación menores que en vacas que no fueron expuestas a esa condición ambiental (Castaño et al., 2014).

Un aumento en la temperatura uterina de $0.5^{\circ} \mathrm{C}$, durante días calurosos, provoca disminución de la tasa de fecundación. En los bovinos, la exposición de novillas a $32^{\circ} \mathrm{C}$ durante 72 horas después de la inseminación artificial, inhibe el desarrollo embrionario, sin embargo, se sabe que el $48 \%$ de las hembras mantenidas $21^{\circ} \mathrm{C}$, pueden quedar gestante sin problema alguno, incluso si el estrés térmico se presenta después de los 10 días posterior al servicio, la fertilidad no es afectada. 
El estrés calórico (EC) tambien actúa directamente sobre el ovocito y la función folicular, comprometiendo la calidad del mismo y promoviendo alteraciones de la dinámica folicular, afectando directamente la fertilidad en vacas lactantes; en estudios se ha demostrado que la fertilidad puede variar según la estación del año, por ejemplo en invierno disminuye cerca del 50\%; en verano $20 \%$ y en el otoño es más baja que en el invierno. Se ha podido observar que en verano el $80 \%$ de los estros pueden ser indetectables. Además, se ha indicado que cuando las temperaturas rectales de los animales aumentan de 38.5 a $40^{\circ} \mathrm{C}$ en 72 horas después del servicio o la inseminación, las tasas de preñez pueden disminuir hasta en el $50 \%$ (Castaño et al., 2014; Ambrose, 1999; Gilad et al., 1993; Ryan et al., 1992) (Figura 1).

Otros estudios en novillas y vacas han indicado que la disminución en la calidad del ovocito en el periodo temprano del posparto, está asociada con balance de energía negativo y las bajas condiciones corporales de los animales, lo cual se expresa en un aumento de embriones subdesarrollados y anormales, teniendo como consecuencia pérdida de embriones en los meses más calurosos del año (Wolfenson et al., 1997).

Varios estudios han indicado que en el ganado bovino, el desarrollo embrionario es altamente sensible a altas temperaturas, entre los primeros tres a 11 días después del servicio; adquiriendo más tolerancia a altas temperaturas a medida que el periodo de gestación avanza. Se sabe que los embriones obtenidos mediante fecundación in vitro (FIV), son más susceptibles al estrés calórico que los obtenidos en condiciones naturales. La mayor pérdida de embriones de bovinos obtenidos de FIV, ocurren antes de los 42 días, cuando las hembras están bajo estrés calórico (Ambrose et al., 1999; Ealy et al., 1994) (figura 1).

Los efectos del EC sobre la preñez se relacionan con la disminución del peso del ternero al nacimiento, alteración de las concentraciones hormonales de la madre y del feto, y reducción de la producción de leche durante el posparto. La disminución en el peso del ternero del orden del $18 \%$ se atribuye a una disminución en las concentraciones de sulfato de estrona (Castaño et al., 2014). 


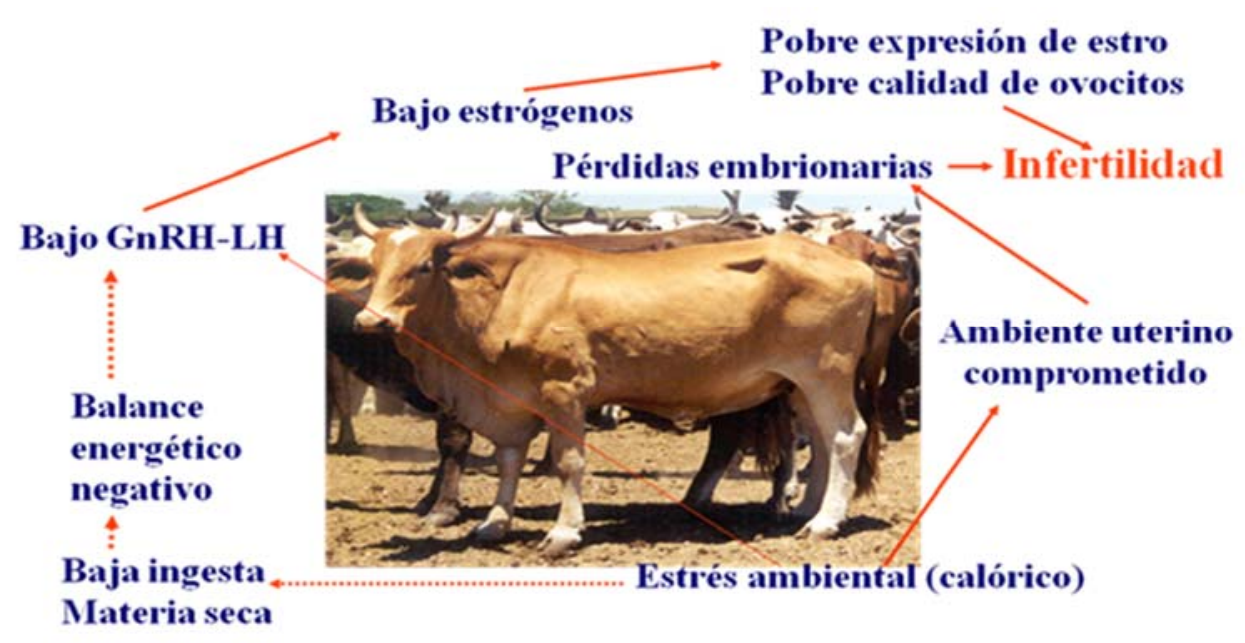

Figura 1. Efecto del estrés calórico en la reproducción

\section{CONCLUSIONES}

Es muy importante tomar ciertas medidas en cuenta para el control del estrés calórico y de esta manera disminuir su efecto sobre la reproducción animal, como son proporcionar un sistema de ventilación que controle la temperatura corporal, implementar rociadores de agua dentro de la producción, proteger a los animales contra las radiaciones solares por medio de sombras o techos apropiados, proporcionar sombra en comederos y bebederos para aumentar el consumo de alimento en animales con estrés calórico, dar a los animales que los necesiten baños por aspersión en las horas más calurosas del día, tratar de tener animales con pelaje blanco, ya que son las que absorben más fácilmente el calor y por lo tanto son menos sensibles al estrés calórico; desarrollar genética adaptada en los animales, ya que pueden ser menos sensibles al estrés calórico; inseminar con semen congelado en época menos calurosa y se debe promover entre los productores las cruzas entre razas de Bos indicus y Bos taurus, esto con el fin de obtener ejemplares F1, que tengan mayor resistencia al calor y las hembras sean mejores productoras de leche.

Las medidas preventivas y de control durante la época de elevadas temperaturas son las siguientes:

La carga animal tiene una gran relación con la capacidad del organismo animal para perder calor, por lo que es importante evitar el hacinamiento para que los animales puedan perder calor de forma eficiente por radiación, convección y conducción, a través del simple hecho de cambiar de postura. También el agua debe permanecer en la sombra lo más fresca posible ya que en las especies con abundantes glándulas sudoríparas en la piel, como por 
ejemplo el caballo, y los bovinos Bos indicus mantiene un mecanismo de enfriamiento eficiente condicionado a que la ingesta de agua sea suficiente. Otra causa importante de hipertermia o estrés calórico es el excesivo trabajo muscular, por lo cual el manejo de los animales en épocas de calor elevado debe ser limitado. También es importante mantener un manejo adecuado del microclima (temperatura, humedad y ventilación) porque puede ayudar a mitigar los efectos del calor en las producciones intensivas. En las producciones extensivas, es importante que se dispongan de árboles y/o sombreaderos en cantidad suficiente, de manera que cada bovino pueda disponer de una protección adecuada de la radiación solar y el calor.

\section{BIBLIOGRAFÍA}

Aitken, R.J., y De Luliis, G.N., 2010. On the possible origins of DNA damage in human spermatozoa, Molecular Human Reproduction, 16, 3-13

Ambrose JD, Drost M, Monson RL, Rutledge JJ, Leibfried-Rutredge ML, Thatcher M-J, Kassa T, Binelli M, Hansen PJ, Chenoweth PJ y Thatcher WW. 1999. Efficacy of timed embryo transfer with fresh and frozen in vitro produced embryos to increase pregnancy rates in heatstresed dairy cattle. J Dairy Sci.; 82 (11): 2369-2376.

Blecha F. 2000. Immunity system response to stress. In: Moberc, G.P. And Merch, Ja. 2000. The Biology Of Animal Stress, Basic Principles And Implications For Animal Welfare. New York, Cabi Publishing: Pp 11-118.

Castaño Faider A., Rugeles P. Clara C., Betancur H. César A. y Ramirez López Camilo J. 2014. Impacto del estrés calórico sobre la actividad reproductiva en bovinos y consideraciones para mitigar sus efectos sobre la reproducción. Biosalud; 13 (2): 84-94.

Chemineau P. 1993. Medio ambiente y reproducción animal. Disponible En: Www.Acontece.Com.Ar/0113.Htm

Coubrough RI. 1985. Stress and fertility. A Review. Onderstepoort J Vet Res.; 52 (3):153-6. Review.

Dobson H y Smith RF. 1995. Stress and reproduction in farm animals. J Reprod. Fertil. Suppl.; 49: 451-61. 
Dobson H y Smith RF. 2000. What is stress, and how does it affect reproduction?. Anim. Reprod. Sci.; 2(60-61):743-52.

Ealy AD, Arechiga CF, Bray DR, Risco CA y Hansen PJ. 1994. Effectiveness of short-term cooling and vitamin e for alleviation of infertility induced by heat stress in dairy cows. J. Dairy Sci.; 77 (12): 3601-7.

Fowler M. E. 2006. Multisystem disorders (stress). In Fowler, Me. And Mikota Sk. Eds. Biology Medicine and Surgery of Elephants. Ames, Iowa. Blackwell Publishing: Pp 243-53.

Fowler M. E. 2008. Restraint and handling domestic animals. Ames: Wiley-Blackwell. Pp: 4568.

Gilad E, Meidan R, Berman A, Graber Y y Wolfenson D. 1993. Effect Of Heat Stress On Tonic And Gnrh-Induced Gonadotrophin Secretion In Relation To Concentration Of Oestradiol In Plasma Of Cyclic Cows. J. Reprod. Fertil. 99 (2): 315-21.

Jung, A. y Schuppe, H.C., 2007. Influence of genital heat stress on semen quality in humans, Andrologia, 39, 203-215

Nezhad, F.S., Lavvaf, A. y Karimi, S., 2013. Effect of heat stress on oxidative reactions in the sheep Sertoli cells, International Journal of Agriculture and Crop Sciences, 6, 833-839

Rutledge JJ. 2001. Use of embryo transfer and IVF to bypass effects of heat stress. Theriogenology; 55 (1): 105-11.

Ryan DP, Blakewood EG, Lynn JW, Munyakazi L y Godke RA. 1992. Effect of heat-stress on bovine embryo development in vitro. J Anim. Sci.; 70 (11): 3490-7.

Sánchez Rodríguez Sergio Hugo. 2007. Respuesta hormonal de los organismos superiores ante el estrés calórico. REDVET; 8 (12): 1-8.

Tremellen, K., 2008. Oxidative stress and male infertility - a clinical perspective. Human Reproduction Update, 14, 243-258

Wechalekar, H., Setchell, BP., Peirce, E.J., Ricci, M., Leigh, C., y Breed, W.G., 2010. Whole body heat exposure induces membrane changes in spermatozoa from the cauda epididymidis of laboratory mice. Asian Journal of Andrology, 12, 591-598 
Wolfenson D, Lew BJ, Thatcher WW, Graber y Meidan R. 1997. Seasonal and acute heat stress effects on steroid production by dominant follicles in cows. Anim. Reprod. Sci.; 47 (1-2): 919.

Yabuta Osorio y Kunio A. 2000. El estrés calórico en el ganado lechero. Disponible En: Http://Fmvz1.Uat.Mx/Investigacion/Memorias/Principa17.Htm

Yokoyama Kano Javier S., Alzina López Alejandro, Farfán Escalante Jorge C. y Valencia Heredia Eduardo R. 2004. Respuestas conductuales termorregulatorias de búsqueda de sombre en bovinos cruzados Bos taurus $x$ Bos indicus criados en la zona costera y oriente del estado de Yucatán. Rev Biomed; 15 (1): 17-26. 